\title{
Influence of myocardial fiber/sheet orientations on left ventricular mechanical contraction
}

\author{
T.S.E. Eriksson ${ }^{\mathrm{a}, \mathrm{b}}$, A.J. Prassl ${ }^{\mathrm{b}}$, G. Plank ${ }^{\mathrm{b}}$, G.A. Holzapfel ${ }^{\mathrm{a}, \mathrm{c}, *}$ \\ ${ }^{a}$ Institute of Biomechanics, Graz University of Technology, Kronesgasse 5-I, A-8010 Graz, Austria \\ ${ }^{b}$ Department of Biophysics, Medical University of Graz, Harrachgasse 21, A-8010 Graz, Austria \\ ${ }^{c}$ Department of Solid Mechanics, School of Engineering Sciences, Royal Institute of Technology (KTH) \\ Osquars Backe 1, SE-100 44 Stockholm, Sweden
}

To appear in

Mathematics and Mechanics of Solids

\begin{abstract}
At any point in space the material properties of the myocardium are characterized as orthotropic, that is, there are three mutually orthogonal axes along which both electrical and mechanical parameters differ. To investigate the role of spatial structural heterogeneity in an orthotropic material, electro-mechanically coupled models of the left ventricle (LV) were used. The implemented models differed in their arrangement of fibers and sheets in the myocardium, but were identical otherwise: (i) a generic homogeneous model, where a rule-based method was applied to assign fiber and sheet orientations, and (ii) a heterogeneous model, where the assignment of the orthotropic tissue structure was based on experimentally obtained fiber/sheet orientations. While both models resulted in pressure-volume loops and metrics of global mechanical function that were qualitatively and quantitatively similar and matched well with experimental data, the predicted deformations were strikingly different between these models, particularly with regard to torsion. Thus, the simulation results strongly suggest that heterogeneous structure properties are playing an important non-negligible role in LV mechanics and, consequently, should be accounted for in computational models.
\end{abstract}

\section{Introduction}

The capability of the heart to efficiently pump blood around the circulatory system is of vital importance. The underlying electro-mechanical function is governed by an interwoven cascade of events that interact across a broad range of spatial and temporal scales. Comprehensive, biophysicallydetailed computer models of these multi-scale and multi-physics phenomena play an important role in a better understanding of integrated electro-mechanical function in health and disease. A key

\footnotetext{
${ }^{*}$ Corresponding author; G.A. Holzapfel, e-mail: hol zapfel@tugraz . at
} 
factor in such models is the accurate representation of the myocardial tissue structure, in particular of the left ventricle (LV), which is the main pumping chamber.

In the LV the prevailing myocyte orientations, usually referred to as fibers, follows a righthanded helical pathway from the endocardium towards the mid-wall, and a left-handed helical pathway from the mid-wall towards the epicardium, see, e.g., [1, 2]. In addition to this transmural change in the inclination angle of the fiber, termed 'fiber rotation', myocytes are bundled and form layers of four to six cells, which are referred to as laminae or sheets. Sheet orientations vary as well, not only transmurally, but also in the apico-basal direction $[2,3]$. At any point in space, the LV is characterized by an orthotropic material with three preferred eigendirections, oriented along fibers, transverse to the fibers but within a sheet, and orthogonal to the sheets.

Orthotropic material properties in the LV influence both the spread of electrical activation and re-polarization and the mechanical response to pressure load and active contraction. Electrically, wavefronts travel fastest along the fibers and slowest in the sheet normal direction, with velocity ratios of roughly $3: 2: 1$ along the three axes [4]. Via electro-mechanical coupling these spatiotemporal patterns of electrical activity trigger active stress transients in the myocytes, which are either modeled as active stresses or strains [5] acting along the orientation of the fibers in a current configuration [6]. The resulting mechanical deformations are then largely determined by both the passive hyperelastic orthotropic properties of the tissue and the generated active stresses.

Many numerical studies of ventricular mechanics use a simple generic rule-based approach to define the fiber and sheet angles, see, e.g., [1, 7-15]. In these studies, the fibers often vary linearly between the endocardium and the epicardium. When orthotropic models are used, the sheet angles either follow the radial direction or also vary linearly between the endocardium and the epicardium. It is with good reason that these studies have chosen such a simple fiber/sheet structure; as it is often difficult to obtain good detailed fiber/sheet data in vivo. However, it has been shown that using fiber/sheet angles that do not vary linearly through the thickness yields more realistic mechanical responses [16, 17].

In the present study we investigate the mechanical effect of models when based on experimentally obtained fiber/sheet orientations and on generic rule-based fiber/sheet orientations, as frequently used in the literature. We compare the two model results and use an invariant-based orthotropic constitutive equation [18]. A weakly coupled electro-mechanical model of the LV is employed where the LV anatomy is approximated as a truncated ellipsoid [9, 19]. The model is equipped with two sets of fiber and sheet arrangements. In the first arrangement, a generic rulebased dataset is used where fiber/sheet angles vary linearly in the transmural direction, as seen previously in, e.g., [1, 7-15]. Several values for the linear change of fiber/sheet angles are examined. In the second arrangement, an experimentally-obtained dataset is used [2], where fiber/sheet angles vary spatially throughout the LV. Simulation results reveal that the generic fiber-sheet setup predicts an incorrect torsion up to five times larger than what is observed experimentally [20, 21], as opposed to an experiment-based orthotropic setup where the torsion is found to be close to the expected range. However, lumped parameter results such as PV loops and cardiac output show only a small difference between the two setups. 


\section{Material and Methods}

\subsection{Electrophysiological modeling}

The spread of electrical activation and re-polarization is described by the mono-domain equation

$$
\beta_{\mathrm{m}} C_{\mathrm{m}} \frac{\partial V_{\mathrm{m}}}{\partial t}+\beta_{\mathrm{m}} I_{\mathrm{ion}}\left(V_{\mathrm{m}}, \boldsymbol{\eta}\right)=\nabla \cdot\left(\mathbf{g}_{\mathrm{m}} \nabla V_{\mathrm{m}}\right)+I_{\mathrm{tr}},
$$

where $\beta_{\mathrm{m}}$ is the membrane surface to volume ratio, $C_{\mathrm{m}}$ is the membrane capacitance, $V_{\mathrm{m}}$ is the transmembrane potential, $I_{\text {ion }}$ is the density of the total ionic current which is a function of $V_{\mathrm{m}}$ and a set of state variables, $\boldsymbol{\eta}, I_{\mathrm{tr}}$ is a transmembrane stimulus current, and $\mathrm{g}_{\mathrm{m}}$ is the mono-domain conductivity tensor given by

$$
\mathbf{g}_{\mathrm{m}}=g_{\mathrm{m}, \mathrm{f}}\left(\mathbf{f}_{0} \otimes \mathbf{f}_{0}\right)+g_{\mathrm{m}, \mathrm{s}}\left(\mathbf{s}_{0} \otimes \mathbf{s}_{0}\right)+g_{\mathrm{m}, \mathrm{n}}\left(\mathbf{n}_{0} \otimes \mathbf{n}_{0}\right),
$$

with the eigenaxes $\mathbf{f}_{0}$ oriented along the fibers, $\mathbf{s}_{0}$ perpendicular to the fibers, but within a laminar sheet, and $\mathbf{n}_{0}$ perpendicular to the sheets, and $\operatorname{diag}\left(g_{\mathrm{m}, \mathrm{f}}, g_{\mathrm{m}, \mathrm{s}}, g_{\mathrm{m}, \mathrm{n}}\right)$ are the corresponding conductivities along the tensor axes. The model by Mahajan et al. [22], as developed for the rabbit ventricular myocytes, is employed to describe cellular dynamics.

\subsection{Active and passive mechanical modeling}

The Cauchy stress tensor $\sigma$ is additively decomposed by

$$
\boldsymbol{\sigma}=\boldsymbol{\sigma}_{\mathrm{p}}+\boldsymbol{\sigma}_{\mathrm{a}}
$$

where $\sigma_{\mathrm{p}}$ refers to the passive stresses that occur in the material during a cardiac cycle due to the intra-ventricular pressure (thereby the myocytes are fully relaxed), and $\sigma_{\mathrm{a}}$ refers to the active stresses intrinsically developed from the contraction of myocytes, when they are appropriately stimulated. Hence, the stress components are assumed to be represented as the sum of two parts.

\subsubsection{Passive stress component}

With regard to the mechanical deformation the myocardium is characterized as an orthotropic material with the eigenaxes $\mathbf{f}_{0}, \mathbf{s}_{0}$ and $\mathbf{n}_{0}$ in the Lagrangian description. Using the multiplicative decomposition of the deformation gradient $\mathbf{F}$, into a volumetric $J^{1 / 3} \mathbf{I}$ and an isochoric $\overline{\mathbf{F}}$ part so that $\mathbf{F}=\left(J^{1 / 3} \mathbf{I}\right) \overline{\mathbf{F}}$, where $J=\operatorname{det} \mathbf{F}>0$ is the volume ratio, the Lagrangian direction vectors are transformed into their isochoric Eulerian counterparts by $\overline{\mathbf{f}}=\overline{\mathbf{F}} \mathbf{f}_{0}, \overline{\mathbf{s}}=\overline{\mathbf{F}} \mathbf{s}_{0}$ and $\overline{\mathbf{n}}=\overline{\mathbf{F}} \mathbf{n}_{0}$. An orthotropic, invariant-based strain-energy function [18] is used for describing the nonlinear passive behavior of the myocardium, which yields a frame-independent stress tensor. The strain-energy function described in [18] is here extended to include the multiplicative decomposition of the deformation gradient. Hence, the strain-energy function $\Psi=U(J)+\bar{\Psi}_{\text {iso }}\left(\bar{I}_{1}\right)+$ $\bar{\Psi}_{\text {aniso }}\left(\bar{I}_{4 \mathrm{f}}, \bar{I}_{4 \mathrm{~s}}, \bar{I}_{8 \mathrm{fs}}\right)$ is separated into a volumetric function $U(J)$ and two volume preserving functions $\bar{\Psi}_{i}, i \in\{$ iso, aniso $\}$, which relate to the isotropic and the anisotropic behavior, respectively. A standard volumetric function $U(J)=\mu_{\mathrm{K}} \ln (J)^{2} / 2$ is used, where the bulk modulus $\mu_{\mathrm{K}}$ serves 
as a penalty parameter for enforcing incompressibility. The volume preserving functions $\bar{\Psi}_{\text {iso }}$ and $\bar{\Psi}_{\text {aniso }}$ are described as

$$
\bar{\Psi}_{\text {iso }}=\frac{a}{2 b}\left\{\exp \left[b\left(\bar{I}_{1}-3\right)\right]-1\right\} \text {, }
$$

and

$$
\bar{\Psi}_{\text {aniso }}=\sum_{i=\mathrm{f}, \mathrm{s}} \frac{a_{i}}{2 b_{i}}\left\{\exp \left[b_{i}\left(\bar{I}_{4 i}-1\right)^{2}\right]-1\right\}+\frac{a_{\mathrm{fs}}}{2 b_{\mathrm{fs}}}\left[\exp \left(b_{\mathrm{fs}} \bar{I}_{8 \mathrm{fs}}^{2}\right)-1\right],
$$

respectively, where in total eight material parameters $\left(a, b, a_{(\mathrm{f}, \mathrm{s}, \mathrm{fs})}\right.$ and $\left.b_{(\mathrm{f}, \mathrm{s}, \mathrm{fs})}\right)$ are needed to describe the isochoric orthotropic material behavior. By using the modified volume-preserving right Cauchy-Green tensor $\overline{\mathbf{C}}=\overline{\mathbf{F}}^{\mathrm{T}} \overline{\mathbf{F}}$, the isochoric invariants in (4) and (5) are defined as $\bar{I}_{1}=$ $\operatorname{tr}(\overline{\mathbf{C}}), \bar{I}_{4 \mathrm{f}}=\mathbf{f}_{0} \cdot\left(\overline{\mathbf{C}} \mathbf{f}_{0}\right), \bar{I}_{4 \mathrm{~s}}=\mathbf{s}_{0} \cdot\left(\overline{\mathbf{C}} \mathbf{s}_{0}\right)$ and $\bar{I}_{8 \mathrm{fs}}=\mathbf{f}_{0} \cdot\left(\overline{\mathbf{C}} \mathbf{s}_{0}\right)$. As shown in [18], necessary conditions on (4) and (5) for material stability are $a, b, a_{(\mathrm{f}, \mathrm{s}, \mathrm{fs})}, b_{(\mathrm{f}, \mathrm{s}, \mathrm{fs})} \geq 0$ and $\bar{I}_{4 \mathrm{f}}, \bar{I}_{4 \mathrm{~s}}>1$. If any of the invariants are less than one, the term containing it is dropped from Eq. (5). In addition, by using $\bar{I}_{4 \mathrm{n}}=\mathbf{n}_{0} \cdot\left(\overline{\mathbf{C n}}_{0}\right)$, it can be shown that $\bar{I}_{4 \mathrm{f}}+\bar{I}_{4 \mathrm{~s}}+\bar{I}_{4 \mathrm{n}}=\bar{I}_{1}$ so that only three invariants are independent; we omit here $\bar{I}_{4 \mathrm{n}}$ from (5) which is the analogue of the expression provided in [18]. The passive Cauchy stress tensor is then given by $\boldsymbol{\sigma}_{\mathrm{p}}=2 J^{-1} \mathbf{F}(\partial \Psi / \partial \mathbf{C}) \mathbf{F}^{\mathrm{T}}$, and therefore

$$
\boldsymbol{\sigma}_{\mathrm{p}}=p_{\mathrm{h}} \mathbf{I}+2 J^{-1}\left[\bar{\psi}_{1} \operatorname{dev} \overline{\mathbf{b}}+\bar{\psi}_{4 \mathrm{f}} \operatorname{dev}(\overline{\mathbf{f}} \otimes \overline{\mathbf{f}})+\bar{\psi}_{4 \mathrm{~s}} \operatorname{dev}(\overline{\mathbf{s}} \otimes \overline{\mathbf{s}})+\frac{1}{2} \bar{\psi}_{8 \mathrm{fs}} \operatorname{dev}(\overline{\mathbf{f}} \otimes \overline{\mathbf{s}}+\overline{\mathbf{s}} \otimes \overline{\mathbf{f}})\right]
$$

where $p_{\mathrm{h}}=\mathrm{d} U(J) / \mathrm{d} J$ is used, $\overline{\mathbf{b}}=\overline{\mathbf{F F}}^{\mathrm{T}}$ is the isochoric modified left Cauchy-Green tensor and $\operatorname{dev}(\bullet)=(\bullet)-(1 / 3)[(\bullet): \mathbf{I}] \mathbf{I}$ is the deviatoric operator in the Eulerian description [23]. Furthermore, in (6), the definitions

$$
\bar{\psi}_{i}=\frac{\partial \Psi}{\partial \bar{I}_{i}}, \quad i=1,4 \mathrm{f}, 4 \mathrm{~s}, 8 \mathrm{fs}
$$

have been used.

\subsubsection{Active stress component}

The active stress tensor $\sigma_{\mathrm{a}}$ is defined as

$$
\boldsymbol{\sigma}_{\mathrm{a}}=J^{-1} S_{\mathrm{a}}(\hat{\mathbf{f}} \otimes \hat{\mathbf{f}}),
$$

where $S_{\mathrm{a}}$ is an active second Piola-Kirchhoff stress component, and $\hat{\mathbf{f}}=\overline{\mathbf{f}} /|\overline{\mathbf{f}}|$ is the normalized fiber direction vector. As a weakly coupled approach is used (see Section 2.5) it is appropriate to calculate the active stress component in the material configuration; the electrical simulations are performed on a static mesh. Following [24], in this study $S_{\mathrm{a}}$ is calculated using the single ordinary differential equation of the form

$$
\dot{S}_{\mathrm{a}}=\epsilon\left(V_{\mathrm{m}}\right)\left(k_{S_{\mathrm{a}}} \Delta V_{\mathrm{m}}-S_{\mathrm{a}}\right),
$$

where $\epsilon\left(V_{\mathrm{m}}\right)$ is a delay function controlling the rate of activation and relaxation of $S_{\mathrm{a}}$, and $k_{S_{\mathrm{a}}}$ regulates the amplitude of $S_{\mathrm{a}}$ as a function of the deviation of the transmembrane potential $V_{\mathrm{m}}$ from the myocyte resting potential $V_{\mathrm{r}}$, i.e. $\Delta V_{\mathrm{m}}=V_{\mathrm{m}}-V_{\mathrm{r}}$. Instead of the Heaviside function proposed in [24], we follow [12] and use a smoother delay function

$$
\epsilon\left(V_{\mathrm{m}}\right)=\epsilon_{0}+\left(\epsilon_{\infty}-\epsilon_{0}\right) \exp \left\{-\exp \left[-\zeta_{\mathrm{r}}\left(V_{\mathrm{m}}-V_{\mathrm{s}}\right)\right]\right\}
$$


where $\epsilon_{0}$ and $\epsilon_{\infty}$ are upper and lower bounds for the transmembrane potential, i.e. for $V_{\mathrm{m}} \ll V_{\mathrm{s}}$ and $V_{\mathrm{m}} \gg V_{\mathrm{s}}$, respectively, where $V_{\mathrm{s}}$ is a given phase shift, and $\zeta_{\mathrm{r}}$ is the transition rate. Note, however, that there is an erratum in the delay function shown in Eq. (23) of [24]. With the choice $\epsilon_{\infty}=10 \epsilon_{0}$ an electro-mechanical delay between an upstroke of the action potential and the peak active stress, as illustrated in Figure 2 in [24], cannot be obtained. In order to reproduce the reported time course of $S_{\mathrm{a}}$ the relation $\epsilon_{\infty}<\epsilon_{0}$ must hold. The authors in [12] have used the original parameter relation, i.e. $\epsilon_{\infty}=10 \epsilon_{0}$, resulting in a delay function that goes from lower to higher values and, thus, to an active stress transient with non-physiologically short electro-mechanical delay (see Figure 3 in [12]). The resulting differences in the shape of $\epsilon\left(V_{\mathrm{m}}\right)$ and the active stress transients are illustrated in Figure 1.

\subsection{Geometry}

A simplified LV geometry is modeled as an ellipsoid truncated at the base using prolate spheroidal coordinates $\xi_{1}, \xi_{2}$ and $\xi_{3}$, see Figure 2. Using the focal position $d_{i}=\sqrt{a_{i}^{2}-b_{i}^{2}}, i \in\{$ endo, epi , where $a_{i}$ and $b_{i}$ are the polar and equatorial axes for the endocardial and epicardial borders, respectively, the prolate spheroidal coordinates may be expressed in a Cartesian coordinate system by $x_{1}=d_{i} \sinh \xi_{1} \sin \xi_{2} \cos \xi_{3}, x_{2}=d_{i} \sinh \xi_{1} \sin \xi_{2} \sin \xi_{3}$ and $x_{3}=d_{i} \cosh \xi_{1} \cos \xi_{2}$. Epicardial and endocardial dimensions are chosen as $a_{\text {epi }}=19.3 \mathrm{~mm}, b_{\text {epi }}=12.7 \mathrm{~mm}$ and $a_{\text {endo }}=18.0 \mathrm{~mm}$, $b_{\text {endo }}=6.9 \mathrm{~mm}$, respectively, to arrive at a good match with an available image-based rabbit LV geometry [25]. The $\xi_{2}$-angle has a maximum value of $120^{\circ}$ at the endocardial surface. When going in the $\xi_{1}$-direction along the epicardial surface the maximum $\xi_{2}$-angle is decreased so that the basal surface remains flat in the global $X_{3}$-direction.

\subsubsection{Model $I$ - Generic fiber-sheet setup}

In model $I$ a generic fiber-sheet setup is implemented. Fibers rotate linearly from $\alpha_{\text {endo }}$ at the endocardial border to $\alpha_{\text {epi }}$ at the epicardial border along the transmural axis $\xi_{1}$, with the inclination angle $\alpha$ measured in the $\left(\xi_{2}, \xi_{3}\right)$ plane, see Figure 2. Similarly, a linear rotation of sheet angles from $\beta_{\text {endo }}$ at the endocardial border to $\beta_{\text {epi }}$ at the epicardial border is imposed in the transmural direction $\xi_{1}$ with the sheet angle $\beta$ measured in the $\left(\xi_{1}, \xi_{2}\right)$ plane, see Figure 2. Since both fiber and sheet orientations vary only along the transmural direction $\xi_{1}$, this setup is homogeneous in the circumferential and longitudinal directions $\xi_{2}$ and $\xi_{3}$. We, therefore, label this setup as homogeneous. Different combinations of fiber/sheet orientations, taken from the existing literature, is examined, see, e.g., [1, 7-15]. The cases examined in this study are as follows:

(i) $\alpha_{\text {endo }}=-60^{\circ}, \alpha_{\text {epi }}=+60^{\circ}, \beta_{\text {endo }}=0^{\circ}, \beta_{\text {epi }}=0^{\circ}$,

(ii) $\alpha_{\text {endo }}=-45^{\circ}, \alpha_{\text {epi }}=+45^{\circ}, \beta_{\text {endo }}=0^{\circ}, \beta_{\text {epi }}=0^{\circ}$,

(iii) $\alpha_{\text {endo }}=-60^{\circ}, \alpha_{\text {epi }}=+85^{\circ}, \beta_{\text {endo }}=0^{\circ}, \beta_{\text {epi }}=0^{\circ}$,

(iv) $\alpha_{\text {endo }}=-60^{\circ}, \alpha_{\text {epi }}=+60^{\circ}, \beta_{\text {endo }}=-85^{\circ}, \beta_{\text {epi }}=+85^{\circ}$,

(v) $\alpha_{\text {endo }}=-45^{\circ}, \alpha_{\text {epi }}=+45^{\circ}, \beta_{\text {endo }}=-85^{\circ}, \beta_{\text {epi }}=+85^{\circ}$,

(vi) $\alpha_{\text {endo }}=-60^{\circ}, \alpha_{\text {epi }}=+85^{\circ}, \beta_{\text {endo }}=-45^{\circ}, \beta_{\text {epi }}=+45^{\circ}$. 
We have here attempted to cover values which are commonly used in the literature, including sheet directions that are aligned in the radial direction $\xi_{1}$ (i.e. $\beta=0^{\circ}$ ) and inclination angles $\alpha$ which lead to a non-symmetry, as seen in the cases (iii) and (vi).

\subsubsection{Model $I I$ - Experimentally-based LV fiber-sheet setup}

To study the influence of structural heterogeneity, a fiber-sheet setup, which is based on experimental data, is implemented in model $I I$. The fiber and sheet orientations are here assigned to fit the diffusion MRI data of Rohmer et al. [2] (Figure 3), which reported fiber and sheet angles in eight different regions corresponding to four circumferential segments of the LV, septal, anterior, lateral and posterior in each of the two apico-basal segments, basal and apical. A linear weighting function is used in between these regions to ensure a smooth transition of fiber and sheet angles. In contrast to model $I$, model $I I$ is spatially heterogeneous in all directions, not only in the transmural direction. We, therefore, label this setup as heterogeneous.

\subsubsection{Torsion}

To analyze the torsion of the LV we define the long axis to be aligned with the main direction of the septal wall and the short axis to be perpendicular to the long axis, and chosen to be close to the endocardial apex, see Figure 6(a). Aligning the centroid of the cavity in the short axis images, taken from end diastolic and end systolic volumes, see Figure 6(c)-(d), the torsion is calculated by tracking the movement of the mesh between these two configurations.

\subsection{Material parameters}

The mono-domain bulk conductivities in Eq. (2) are chosen to be $g_{\mathrm{m}, \mathrm{f}}=0.204 \mathrm{Sm}^{-1}, g_{\mathrm{m}, \mathrm{s}}=$ $0.102 \mathrm{Sm}^{-1}$ and $g_{\mathrm{m}, \mathrm{n}}=0.037 \mathrm{Sm}^{-1}$, which lead to conduction velocities of $0.6 \mathrm{~ms}^{-1}, 0.4 \mathrm{~ms}^{-1}$ and $0.2 \mathrm{~ms}^{-1}$ along the principal tensor axes. Standard values of $C_{\mathrm{m}}=1 \mu \mathrm{F} \mathrm{cm}^{-2}$ and $\beta_{\mathrm{m}}=1400 \mathrm{~cm}^{-1}$ are chosen for the membrane capacitance and the membrane surface to volume ratio, respectively.

Mechanical material parameters are summarized in Table 1. Passive material parameters are adapted from [18] where the constitutive model was fitted to experimental data reported by Dokos et al. [26]. Active material parameters are in part adapted from [24] and in part to achieve an electro-mechanical delay of $110 \mathrm{~ms}$ between the peak action potential and the peak active stress [27], which leads the parameter relation of the delay function to be $\epsilon_{\infty}=\epsilon_{0} / 10$. The pressure parameters (see Section 2.6.2) are tuned such that realistic pressure-volume loops are obtained, and to keep the pressure calculations numerically stable.

\subsection{Numerical solution}

The finite element method is employed for the spatial discretization of the mono-domain equation (1). Two overlapping finite element meshes of the same ellipsoidal LV geometry are generated, a fully structured coarser hexahedral mesh for solving the mechanics, and a fully unstructured hybrid mesh with an average resolution of $\sim 200 \mu \mathrm{m}$, using an image-based mesh generation technique [28], as implemented in the commercial mesh generator Tarantula (CAE Software Solution, 
Eggenberg, Austria) for solving the electrics. In both meshes, fiber and sheet orientations are interpolated onto the barycenters of the finite elements. The mechanical mesh consists of 5406 nodes and 5310 hexahedral mixed $Q 1 / P 0$ finite elements (see, e.g., [29]), whereas the electrical mesh consists of 1054146 nodes and 1201507 hybrid finite elements using linear weighting functions [30].

Using overlapping grids of different resolutions is a natural choice when considering the vastly different constraints imposed by the physics of the electrical and the mechanical problems. Electrical transients are fast and act on time scales in the $\mu$ s range, which translates into steep depolarization wavefronts of small spatial extent in the sub-millimeter range, thus necessitating the use of fine spatial resolutions $\ll 250 \mu \mathrm{m}$ to compute solutions with reasonable accuracy. On the other hand, mechanical processes tend to occur at larger space and slower time scales, and, thus, coarser spatial discretizations can be used. In the weakly coupled approach applied in this study, Eq. (1) is solved on the electrical mesh first, and electrical quantities required for computing the active stress transients, i.e. $V_{\mathrm{m}}$, are then transferred to the integration points of the mechanical mesh. This means that the electro-mechanical coupling is robust and insensitive to any coupling parameters.

Temporal discretization of the mono-domain equations relies on an implicit-explicit (IMEX) scheme where the diffusion term is treated implicitly and the reaction term explicitly, using a time step of $20 \mu \mathrm{s}$. The linear system is solved in parallel by employing a block Jacobi pre-conditioner with an iterative Conjugate Gradient solver, using an Incomplete LU (ILU(0)) factorization as a sub-block pre-conditioner [31]. The system of ODEs in the model by Mahajan et al. [22] is solved using the Rush-Larsen method [32] with several optimizations [10]. The Cardiac Arrhythmia Research Package (CARP) [33], which is built on top of the MPI-based library PETSc [31], is employed to solve Eq. (1). Numerical aspects have been described in detail elsewhere [34]. The equations (3) together with (6) and (8) are implemented and solved using the finite element software FEAP [35]. Both grids are partitioned for parallel execution using parMETIS [36].

\subsection{Initial values and boundary conditions}

\subsubsection{Electrics}

An initial state vector is computed for the model by Mahajan et al. [22] by pacing a single cell at a pacing cycle length of $350 \mathrm{~ms}$ until arriving at a stable limit cycle. The state vector $\boldsymbol{\eta}$ is then used to populate the LV model. Transmembrane current injection applied to the endocardial surface at $t=0 \mathrm{~ms}$ initiates a transmural activation wavefront. With this protocol, the whole endocardium activates synchronously. Note that we have not included the Purkinje system. An inclusion would lead to a delay of the electrical propagation (especially in the longitudinal direction) within the myocardium. Here $350 \mathrm{~ms}$ of activity is simulated to cover one full depolarization and re-polarization cycle throughout the entire LV.

\subsubsection{Mechanics, circulatory components and pressure-volume loops}

All finite elements on the endocardial surface of the LV are subjected to a follower pressure load $p$. Displacement boundary conditions (BCs) are imposed over the entire base of the LV, i.e. where $\xi_{2}=\xi_{2 \max }$ for all $\xi_{3}$ and $\xi_{1}$ (the subindex max denotes the maximum coordinate value), preventing any movement in the $\xi_{2}$-direction. Additional displacement $\mathrm{BCs}$ are imposed on a subset of nodes 
which are located in the basal plane along the epicardial surface, i.e. where $\xi_{1}=\xi_{1 \max }, \xi_{2}=\xi_{2 \max }$ and for all $\xi_{3}$, to restrict the movement in the $\xi_{3}$-direction. These BCs are summarized in Table 2.

The endocardial pressure load $p$ is calculated in five consecutive steps (i)-(v): (i) initialization phase with linear increase in $p$ to the end diastolic pressure (EDP); before the electrical activation has started, the pressure increases first linearly from 0 to $20 \mathrm{mmHg}$, which is considered the EDP [37]. (ii) Isochoric LV contraction phase: electrical activation starts the isochoric contraction phase where both mitral and aortic valves are closed. Following [38] this is implemented by using the iteration

$$
p_{n+1}=p_{n}+\left(V_{n+1}-V_{n}\right) / C_{\mathrm{p}}
$$

where $C_{\mathrm{p}}$ serves as a penalty parameter that is chosen to give a robust convergence. The iteration is considered to be converged using the criterion $\left\|V_{n+1}-V_{n}\right\| /\left\|V_{n}\right\|<0.01$, which is achieved in only a few iterations by choosing an appropriate value for $C_{\mathrm{p}}$. (iii) Ejection phase where $p$ reaches $95 \mathrm{mmHg}$ [37]. The ejection phase starts in response to the opening of the aortic valve. The pressure is modeled using a two element Windkessel model described as

$$
C \frac{\mathrm{d} p}{\mathrm{~d} t}+\frac{p}{R}=-\frac{\mathrm{d} V}{\mathrm{~d} t}
$$

where $C$ and $R$ relate to the arterial compliance and resistance, respectively. (iv) Isochoric $\mathrm{LV}$ relaxation phase: when $\mathrm{d} V / \mathrm{d} t$ becomes positive (reversed blood flow), the aortic valve closes. The pressure at the instant of flow reversal is considered the end systolic pressure that starts isochoric relaxation, again modeled by using the pressure iteration given through Eq. (11). (v) Filling phase with linear pressure increase to EDP: finally, when $p$ dropped to $12.5 \mathrm{mmHg}$ [37], the mitral valve opens and passive filling starts. Again, this phase is modeled as a linear increase of $p$ up to EDP.

The specific parameters $C, R$ and $C_{\mathrm{p}}$ used in the subsequent analyses are provided in Table 1.

\subsection{Lumped parameters}

The average thickness of the LV wall is calculated using the Gauss divergence theorem. Defining $h_{\mathrm{ed}}$ and $h_{\mathrm{es}}$ as the wall thickness corresponding to end diastolic volume (EDV) and the end systolic volume (ESV), respectively, the fractional thickening $h_{\mathrm{f}}$ is calculated as $h_{\mathrm{f}}=100 \cdot\left(h_{\mathrm{es}}-h_{\mathrm{ed}}\right) / h_{\mathrm{ed}}$. As a coarse measure for examining incompressibility the fractional change in the myocardial volume, say $V_{\mathrm{f}}$, is calculated as $V_{\mathrm{f}}(t)=100 \cdot\left(V_{\text {wall }}(t)-V_{\text {wall }}^{0}\right) / V_{\text {wall }}^{0}$, where $V_{\text {wall }}(t)$ and $V_{\text {wall }}^{0}$ are the volumes of the myocardial wall at time $t$ and in the initial unloaded configuration, respectively. The largest change in the volume fraction $V_{\mathrm{f}}(t)$ is denoted by $V_{\mathrm{f}} \max$, which may be used as an approximate metric to gauge how well the incompressibility constraint is enforced in the simulation. Furthermore, stroke volume $\mathrm{SV}$ is defined as $\mathrm{SV}=\mathrm{EDV}-\mathrm{ESV}$, cardiac output $\mathrm{CO}$ is defined as $\mathrm{CO}=\mathrm{SV} \cdot \mathrm{HR}$, where $\mathrm{HR}$ is the heart rate, and the ejection fraction $\mathrm{EF}$ is defined as $\mathrm{EF}=100 \cdot \mathrm{SV} / \mathrm{EDV}$. Although only one heart beat is simulated, $\mathrm{HR}$ is assumed to follow the simulated activity time of $350 \mathrm{~ms}$, giving a HR of about 171 beats per minute. 


\section{Results}

\subsection{Validation of the implementation}

To test the numerical framework, an analytical test case is designed and explored. A unit myocardium tissue cube with orthogonal material directions is considered. The cube has the fiber, sheet and sheet-normal directions along the global coordinate system $(X, Y, Z)$, and the material directions are according to $\left[\mathbf{f}_{0}\right]=[1,0,0]^{\mathrm{T}},\left[\mathbf{s}_{0}\right]=[0,1,0]^{\mathrm{T}}$ and $\left[\mathbf{n}_{0}\right]=[0,0,1]^{\mathrm{T}}$, as shown in Figure 4(a). The cube can freely deform, and it is subjected to an active stress $S_{\mathrm{a}}$ resulting in a contraction in the global $X$-direction, i.e. the fiber direction. The material is assumed to be incompressible and, therefore, the cube must expand in the global $Y$ and $Z$-directions. Thus, the corresponding deformation gradient is $\mathbf{F}=\operatorname{diag}\left(\lambda_{\mathrm{f}}, \lambda_{\mathrm{s}}, \lambda_{\mathrm{n}}\right)$, where the stretch $\lambda_{\mathrm{f}}$ in the fiber direction is $\lambda_{\mathrm{f}}<1$ and the stretches in the sheet and sheet-normal directions are $\lambda_{\mathrm{s}}>1$ and $\lambda_{\mathrm{n}}>1$, respectively. As the unit cube is free to deform, the stress at equilibrium is zero for all components of the Cauchy stress matrix and the Lagrange multiplier $p_{\mathrm{h}}$ is readily determined from the relation $\sigma_{33}=0$. Using the condition of a volume preserving incompressible material $(J=\operatorname{det} \mathbf{F}=1)$, the following nonlinear system of equations may be obtained as

$$
\begin{aligned}
& \sigma_{11}=2 \bar{\psi}_{1}\left(\lambda_{\mathrm{f}}^{2}-\lambda_{\mathrm{n}}^{2}\right)+2 \bar{\psi}_{4 \mathrm{f}} \lambda_{\mathrm{f}}^{2}+S_{\mathrm{a}}=0, \\
& \sigma_{22}=2 \bar{\psi}_{1}\left(\lambda_{\mathrm{s}}^{2}-\lambda_{\mathrm{n}}^{2}\right)+2 \bar{\psi}_{4 \mathrm{~s}} \lambda_{\mathrm{s}}^{2}=0 \\
& \lambda_{\mathrm{f}} \lambda_{\mathrm{s}} \lambda_{\mathrm{n}}=1
\end{aligned}
$$

Keeping in mind that $\bar{\psi}_{4 \mathrm{f}}$ vanishes if $\lambda_{\mathrm{f}}<1$ (see [18]), which is always the case in this particular example, the system of Eqs. (13)-(15) can be solved for $\lambda_{\mathrm{f}}, \lambda_{\mathrm{s}}$ and $\lambda_{\mathrm{n}}$ for a given value of the active stress $S_{\mathrm{a}}$. This system is solved using the MATLAB function fsolve() (Matlab R2012a, The Mathworks, Nantucket, USA). The material parameters used are taken from Table 1. The same problem is solved with FEAP, using $Q 1 / P 0$ finite elements, and the resulting stretches are compared to the analytical solution for several values of the active stress $S_{\mathrm{a}}$ computed using a linear increase of the transmembrane potential $V_{\mathrm{m}}$ from the resting potential $V_{\mathrm{m}}=V_{\mathrm{r}}$ to $V_{\mathrm{m}}=+50 \mathrm{mV}$. The results are summarized in Figure 4(b) and show a good agreement between the analytical result and the numerical solution; the different stretches in the figure are abbreviated by $\lambda$.

\subsection{Effect of fiber/sheet arrangement upon mechanical contraction}

To study the effect of the arrangement of fibers and laminae, pressure-volume (PV) loops are computed for a full cardiac cycle by using the models $I$ and $I I$. Both models are subjected to the same stimulation protocol and boundary conditions; the only difference is the fiber and sheet arrangement.

The PV loops predicted by the models $I$ and $I I$ are in good agreement with those observed experimentally [37]. Figure 5(a) shows a comparison between model $I I$ and experimental data. Volume transients for this comparison are normalized to account for the difference in the initial LV volumes. Figure 5(b) compares the PV loops predicted by model $I$ (case (i), Section 2.3.1, chosen as a representative case for the generic distributions), and model $I I$. Overall, both models predict very similar PV loops. The only minor difference is that model $I I$ exhibits a noticeably larger EDV while no significant differences are found in ESV. Moreover, no major differences are observed in 
other lumped metrics that characterize the mechanical performance globally. A comparison of the parameters $h_{\mathrm{f}}, V_{\mathrm{f}}^{\max }, \mathrm{HR}, \mathrm{SV}, \mathrm{EF}$ and $\mathrm{CO}$ is summarized in Table 3, again using case (i), Section 2.3.1, as a representative case for model $I$. The stroke volume SV is slightly lower with model $I$, and thus, since HR is the same, CO is lower too. The relatively small change in the volume fraction $V_{\mathrm{f}}^{\max }$ of the wall indicates that the chosen bulk modulus is large enough to enforce the prescribed incompressibility constraint.

While PV loops and some other metrics of the global mechanical function are qualitatively and quantitatively similar, in several other aspects the model predictions are strikingly different. In the model $I$ the apex moves only along the apico-basal direction, aligned with the global $X_{3}$-direction in our setup, whereas in the model $I I$ a significant shift of the apex towards the septal wall occurs, see Figure 6(b). A significant quantitative difference is observed with regard to torsion. While the average torsion, measured in the short axis plane, predicted by model $I I$ is $4.5^{\circ}$ (Figure 6(c)), a vastly larger torsion is predicted by the generic fiber and sheet setups used in model $I$, which results in, e.g., a torsion of $51^{\circ}$ for case (i), Section 2.3.1 (see Figure 6(d)). A summary of the torsion which results from all generic fiber/sheet setups is shown in Table 4, where the values range from $33-72^{\circ}$. The radial contractions from EDV to ESV, i.e. the local changes of the endocardium and epicardium in the radial direction visible in Figure 6(c)-(d), are analyzed along the septallateral and posterior-anterior directions. In both models $I$ and $I I$, this contraction is quite similar and match experimental data quite well [39], except in the septal-lateral direction at the epicardial border where model $I I$ is significantly closer to the experimental values than model $I$ (all cases).

A further fundamental difference is observed with regard to the first principal stress $\sigma_{I}$. Model $I$ predicts a higher stress in the mid-myocardial band, whilst model $I I$ predicts a gradient of stress from lower to higher transmurally from endocardium to epicardium. This is illustrated in Figure 7, which visualizes $\sigma_{I}$ in a cross section through the septal and lateral wall, again using case (i), as a representative case for model $I$.

\section{Discussion}

A computational model of LV electro-mechanics has been used to investigate the influence of spatial heterogeneity of the structural (orthotropic) components on mechanical contraction. Two models $(I$ and $I I)$ are designed so that they differed only in terms of the fiber and sheet arrangements. As in virtually all recent modeling studies of ventricular electro-mechanics, see, e.g., [6, 9, 24], several simplifying assumptions are made, which may result in model predictions that deviate from experimental observations in one or the other aspect. While these shortcomings warrant caution when drawing general physiological conclusions, it is possible to study whether a given factor is a relevant contributor to the overall response of the system or not.

Although the notion that tissue orthotropy and its spatial structural heterogeneity may play an important role in ventricular mechanics is widely accepted [40], most organ-scale modeling studies reported in the literature refrained from accounting the inherent heterogeneity. To the best of our knowledge, there are only a handful of studies that model the LV as an orthotropic material, see [18] for a review. In fact, most of the modeling studies represent both electrophysiology and mechanics of the ventricles as transversely isotropic materials and, thus, ignore the influence of orthotropy. One reason for this may be the inherent difficulty in the measurements of electrical conductivity and mechanical stress, which has led to an ongoing debate on the 'exact' ratios be- 
tween the eigenvalues and the material parameters along the axes $\mathbf{f}_{0}, \mathbf{s}_{0}$ and $\mathbf{n}_{0}$. However, due to the convincing evidence provided by various studies using histological [41-43] and MRI-based imaging techniques $[2,44]$, and measuring the mechanical properties of the passive ventricular myocardium [26], there is a broad consensus that electrical and mechanical properties of cardiac tissue are best characterized as orthotropic.

In this work we studied the relative influence of heterogeneous orthotropic fiber/sheet structures on mechanical contraction. While the predictions of both models (homogeneous and heterogeneous) are similar and matched fairly well with the experimental data in terms of most lumped system parameters such as PV loops, strikingly different mechanical responses are observed in several other aspects. Most notably, this is the case with torsion, which turns out to be significantly smaller when the structure is considered as heterogeneous.

The simulation results strongly suggest that heterogeneity of the fiber/sheet structure plays an important (non-negligible) role in the mechanical LV contraction and, consequently, should be accounted for in computational models.

\subsection{Effects of structural heterogeneity}

Simulations using model $I$ predicted a torsion which is much higher than the average torsion of $4.5^{\circ}$ predicted by model $I I$. For example, case (i) (Section 2.3.1), predicted an end systolic torsion of $51^{\circ}$. Experimental measurements suggest that the end systolic torsion is in the range of $\sim 8$ $12^{\circ}[20,21]$; hence model $I I$ slightly underestimates torsion whereas model $I$ led to a significant overestimation.

When compared to physiological measurements both models suffered from shortcomings. The pronounced torsion predicted by model $I$ may be attributed to the simplified representation of the LV geometry and the absence of the right ventricle, which would likely restrain torsion significantly, particularly in the septal region. On the other hand, despite the more realistic end systolic torsion in model $I I$, it led to some non-physiological predictions that are not present in model $I$. In particular, model II predicted a longitudinal elongation of the LV, which is in contradiction to the longitudinal shortening, as it is observed in experiments [45]. Potential reasons are: (i) the fiber angles according to Rohmer et al. [2], as used in the model $I I$, tend to be smaller than those observed in histological studies [1] upon which most approaches are built. Thus, due to the reduced fiber component along the long axis of the LV the maximum active stress generated, regulated by the parameter $k_{S_{\mathrm{a}}}$, was not sufficiently large and the cavitary pressure of the LV may have been the dominating factor; (ii) the mapping of orthotropic data from an anatomically realistic model onto a simplified ellipsoidal model may have led to distortions due to the geometrical differences between an idealized ellipsoid and a real ventricular geometry. This difficulty could be circumvented by using a dataset that provides both anatomical accurate representations of the ventricles obtained via high-resolution MRI, and structural information acquired by, e.g., diffusion tensor MRI. A further potential discrepancy stems from the fact that the complex trabeculation of the endocardium remains unaccounted for in our model, a limitation that is shared with all other modeling studies on ventricular mechanics; (iii) the mean values as presented in [2] are used and they are linearly interpolated, however, the noise and variance in these data are big. The data were averaged over large sectors of the ventricles - more detailed data on a per voxel base is not available.

A further difference between the models is the first principal stress $\sigma_{I}$, as illustrated in Figure 7. This seems to be an indirect effect of the reduced torsion in the model $I I$, which, in turn, attenuated 
the influence of the applied boundary conditions.

Stroke volume SV, ejection fraction EF and cardiac output CO are all close to experimentally obtained values, as seen in Table 3. Thus, by using these lumped material parameters and the PV loops, it is only possible to partially differentiate between, or determine the accuracy of, the models given the large variations seen in, e.g., the torsion. The discrepancy between experimentally observed and simulated values for the compressibility of wall volume $V_{\mathrm{f}}^{\max }$ is explained by the fact that ventricular vascularization is not represented in the model. While myocardial tissue is nearly incompressible and, therefore, it is modeled as an incompressible material, the ventricles as a whole are not since the wall volume reduces during systole due to blood being squeezed out of the coronary arteries [46].

\subsection{Limitations of the study}

In our models we have made a few simplifying assumptions and these have to be considered when interpreting the reported results. One limitation is the use of a stylized geometry in which the LV anatomy is approximated by an ellipsoidal shape. Further, a mono-ventricular, not a bi-ventricular geometry is used, thus, the LV is modeled in absence of the right ventricle. Apart from the simplified representation of the macro-anatomy, the complex structure of the ventricular endocardium, consisting of structures such as trabeculae and papillary muscles, is approximated by a smooth endocardial surface. Unlike in electrophysiological modeling studies, where these structures are included in models [47], there have not been any reports yet in the mechanical modeling. Most likely this is due the significant increase in mesh density required to resolve these finer anatomical details, which entails a substantial increase in computational costs [48]. Considering the limited performance of current solver techniques used in modeling ventricular mechanics it suggests that this is not feasible yet.

In the literature a simplified phenomenological model of electro-mechanical coupling is proposed where the generated active stress depends only on the transmembrane potential [12, 24], ignoring all major physiological factors implicated in the process of active stress generation such as calcium transients, the interactions of calcium with myofilaments and metabolic aspects as well as length and velocity dependencies [49]. Finally, as in most previous studies [14, 19, 38], the electrical and mechanical models are weakly coupled. While strongly coupled approaches are likely to be key when subtle electro-mechanical effects have to be captured, most modeling studies opted for implementing a weak coupling approach. This is mainly due to the increased complexity of computation schemes and potential problems with numerical instabilities, although those issues are being addressed [6].

In this study, we have focused on the heterogeneity of the myocardial structure components. Another factor which may alter the simulation results significantly is a heterogeneity of the myocardial material parameters. Such varying material properties, both transmurally, longitudinally and circumferentially could be included in a future study. However, due to the lack of experimental data available on regional-specific myocardial material properties such a study is not feasible at the moment. We have tried to use data from only one species, namely the rabbit. However, due to the limited experimental data available that was not always possible. For example, it was necessary to use the passive material parameters from a porcine myocardium [26]. However, this limitation does not affect the conclusions drawn from varying the fiber/sheet angles. Despite the numerous limitations, which are shared with many contemporary modeling studies on cardiac electro-mechanics, 
the relative difference when using rule-based versus experiment-based data on fiber and sheet arrangements is a clear indication that heterogeneity in the orthotropic structure property is a relevant factor and, as such, it should be accounted for in computational models of ventricular mechanics.

\section{Acknowledgement}

This project was partly supported by the SFB Research Center 'Mathematical Optimization and Applications in Biomedical Sciences', project F3210-N18, granted by the Austrian Science Fund. This support is gratefully acknowledged.

\section{References}

[1] D. D. Streeter, Jr., H. M. Spotnitz, D. P. Patel, J. Ross, Jr., and E. H. Sonnenblick. Fibre orientation in the canine left ventricle during diastole and systole. Circ. Res., 24:339-347, 1969.

[2] D. Rohmer, A. Sitek, and G. T. Gullberg. Reconstruction and visualization of fiber and laminar structure in the normal human heart from ex vivo diffusion tensor magnetic resonance imaging (DTMRI) data. Invest. Radiol., 42:777-789, 2007.

[3] A. A. Young, I. J. Legrice, M. A. Young, and B. H. Smaill. Extended confocal microscopy of myocardial laminae and collagen network. J Microsc., 192:139-150, 1998.

[4] M. J. Bishop and G. Plank. Representing cardiac bidomain bath-loading effects by an augmented monodomain approach: application to complex ventricular models. IEEE T. Biomed. Eng., 58:1066-1075, 2011.

[5] D. Ambrosi and S. Pezzuto. Active stress vs. active strain in mechanobiology: Constitutive issues. J. Elast., 107:199-212, 2012.

[6] S. A. Niederer and N. P. Smith. An improved numerical method for strong coupling of excitation and contraction models in the heart. Prog. Biophys. Mol. Biol., 96:90-111, 2008.

[7] I. J. LeGrice, P. J. Hunter, A. Young, and B. H. Smaill. The architecture of the heart: A data-based model. Philos. T. Roy. Soc. A, 359:1217-1232, 2001.

[8] E. W. Remme, P. J. Hunter, O. Smiseth, C. Stevens, S. I. Rabben, H. Skulstad, and B. B. Angelsen. Development of an in vivo method for determining material properties of passive myocardium. J. Biomech., 37:669-678, 2004.

[9] D. Nickerson, N. Smith, and P. Hunter. New development in a strongly coupled cardiac electromechanical model. Eurospace, 7:S118-S127, 2005.

[10] G. Plank, L. Zhou, J. L. Greenstein, S. Cortassa, R. L. Winslow, B. O’Rourke, and N. A. Trayanova. From mitochondrial ion channels to arrhythmias in the heart: computational techniques to bridge the spatio-temporal scales. Philos. Transact. A Math. Phys. Eng. Sci., 366:3381-3409, 2008. 
[11] S. A. Niederer and N. P. Smith. The role of the Frank-Starling law in the transduction of cellular work to whole organ pump function: a computational modeling analysis. PLoS Comput. Biol., 5:e1000371, 2009.

[12] S. Göktepe and E. Kuhl. Electromechanics of the heart: a unified approach to the strongly coupled excitation-contraction problem. Comput. Mech., 45:227-243, 2010.

[13] S. Göktepe, O. J. Abilez, and E. Kuhl. A generic approach towards finite growth with examples of athlete's heart, cardiac dilation, and cardiac wall thickening. J. Mech. Phys. Solids, 58:1661-1680, 2010.

[14] S. A. Niederer, G. Plank, P. Chinchapatnam, M. Ginks, P. Lamata, K. S. Rhode, C. A. Rinaldi, R. Razavi, and N. P. Smith. Length-dependent tension in the failing heart and the efficacy of cardiac resynchronization therapy. Cardiovasc. Res., 89:336-343, 2011.

[15] S. Rossi, R. Ruiz-Baier, L. F. Pavarino, and A. Quarteroni. Orthotropic active strain models for the numerical simulation of cardiac biomechanics. Int. J. Numer. Method. Biomed. Eng., 28:761-788, 2012.

[16] P. H. M. Bovendeerd, T. Arts, J. M. Huyghe, D. H. van Campen, and R. S. Reneman. Dependence of local left ventricular wall mechanics on myocardial fiber orientation: A model study. J. Biomech., 25:1129-1140, 1992.

[17] T. P. Usyk, R. Mazhari, and A. D. McCulloch. Effect of laminar orthotropic myofiber architecture on regional stress and strain in the canine left ventricle. J. Elasticity, 61:143-164, 2000 .

[18] G. A. Holzapfel and R. W. Ogden. Constitutive modelling of passive myocardium: a structurally based framework for material characterization. Philos. T. Roy. Soc. A, 367:3445-3475, 2009.

[19] R. C. P. Kerckhoffs, P. H. M. Bovendeerd, J. C. S. Kotte, F. W. Prinzen, K. Smits, and T. Arts. Homogeneity of cardiac contraction despite physiological asynchrony of depolarization: A model study. Ann. Biomed. Eng., 31:536-547, 2003.

[20] M. Takeuchi, H. Nakai, M. Kokumai, T. Nishikage, S. Otani, and R. M. Lang. Age-related changes in left ventricular twist assessed by two-dimensional speckle-tracking imaging. $J$. Am. Soc. Echocardiogr., 19:1077-1084, 2006.

[21] F. Carreras, J. Garcia-Barnes, D. Gil, S. Pujadas, C. H. Li, R. Suarez-Arias, R. Leta, X. Alomar, M. Ballester, and G. Pons-Llado. Left ventricular torsion and longitudinal shortening: two fundamental components of myocardial mechanics assessed by tagged cine-MRI in normal subjects. Int. J. Cardiovasc. Imaging, 16:1-12, 2011.

[22] A. Mahajan, Y. Shiferaw, D. Sato, A. Baher, R. Olcese, L. H. Xie, M. J. Yang, P. S. Chen, J. G. Restrepo, A. Karma, A. Garfinkel, Z. Qu, and J. N. Weiss. A rabbit ventricular action potential model replicating cardiac dynamics at rapid heart rates. Biophys. J., 94:392-410, 2008. 
[23] G. A. Holzapfel. Nonlinear Solid Mechanics. A Continuum Approach for Engineering. John Wiley \& Sons, Chichester, 2000.

[24] M. P. Nash and A. V. Panfilov. Electromechanical model of excitable tissue to study reentrant cardiac arrhythmias. Prog. Biophys. Mol. Biol., 85:501-522, 2004.

[25] F. J. Vetter and A. D. McCulloch. Three-dimensional analysis of regional cardiac function: A model of the rabbit ventricular anatomy. Prog. Biophys. Mol. Biol., 69:157-183, 1998.

[26] S. Dokos, B. H. Smaill, A. A. Young, and I. J. LeGrice. Shear properties of passive ventricular myocardium. Am. J. Physiol., 283:H2650-H2659, 2002.

[27] M. M. Monasky, K. D. Varian, J. P. Davis, and P. M. Janssen. Dissociation of force decline from calcium decline by preload in isolated rabbit myocardium. Pflügers Arch. - Eur. J. Physiol., 456:267-276, 2008.

[28] A. J. Prassl, F. Kickinger, H. Ahammer, V. Grau, J. E. Schneider, E. Hofer, E. J. Vigmond, N. A. Trayanova, and G. Plank. Automatically generated, anatomically accurate meshes for cardiac electrophysiology problems. IEEE T. Biomed. Eng., 56:1318-1330, 2009.

[29] G. A. Holzapfel. Structural and numerical models for the (visco)elastic response of arterial walls with residual stresses. In G. A. Holzapfel and R. W. Ogden, editors, Biomechanics of Soft Tissue in Cardiovascular Systems, pages 109-184, Wien, New York, 2003. SpringerVerlag. CISM Courses and Lectures no. 441.

[30] B. M. Rocha, F. Kickinger, A. J. Prassl, G. Haase, E. J. Vigmond, R. Weber dos Santos, S. Zaglmayr, and G. Plank. A macro finite-element formulation for cardiac electrophysiology simulations using hybrid unstructured grids. IEEE T. Biomed. Eng., 58:1055-1065, 2011.

[31] S. Balay, J. Brown, K. Buschelman, V. Eijkhout, W. D. Gropp, D. Kaushik, M. G. Knepley, L. Curfman McInnes, B. F. Smith, and H. Zhang. PETSc users manual. Technical report, Argonne National Laboratory, 2010.

[32] S. Rush and H. Larsen. A practical algorithm for solving dynamic membrane equations. IEEE T. Biomed. Eng., 25:389-392, 1978.

[33] E. J. Vigmond, M. Hughes, G. Plank, and L. J. Leon. Computational tools for modeling electrical activity in cardiac tissue. J. Electrocardiol., 36 Suppl:69-74, 2003.

[34] E. J. Vigmond, R. Weber dos Santos, A. J. Prassl, M. Deo, and G. Plank. Solvers for the cardiac bidomain equations. Prog. Biophys. Mol. Biol., 96:3-18, 2008.

[35] R. L. Taylor. FEAP - A Finite Element Analysis Program, Version 8.2 User Manual. University of California at Berkeley, Berkeley, California, 2008.

[36] G. Karypis, K. Schloegel, and V. Kumar. ParMETIS: Parallel Graph Partitioning and Sparse Matrix Ordering Library. University of Minnesota, Minneapolis, MN, Version 3.1, 2003. URL http://glaros.dtc.umn.edu/gkhome/metis/parmetis/overview. 
[37] C. F. Royse and A. G. Royse. The myocardial and vascular effects of bupivacaine, levobupivacaine, and ropivacaine using pressure volume loops. Anesth. Analg., 101:679-687, 2005.

[38] T. P. Usyk, I. J. LeGrice, and A. D. McCulloch. Computational model of three-dimensional cardiac electromechanics. Comput. Visual. Sci., 4:249-257, 2002.

[39] J. Parkinson, A. Brass, G. Canova, and Y. Brechet. The mechanical properties of simulated collagen fibrils. J. Biomech., 30:549-554, 1997.

[40] D. A. Nordsletten, S. A. Niederer, M. P. Nash, P. J. Hunter, and N. P. Smith. Coupling multi-physics models to cardiac mechanics. Prog. Biophys. Mol. Biol., 104:77-88, 2011.

[41] I. J. LeGrice, B. H. Smaill, L. Z. Chai, S. G. Edgar, J. B. Gavin, and P. J. Hunter. Laminar structure of the heart: Ventricular myocyte arrangement and connective tissue architecture in the dog. Am. J. Physiol. Heart Circ. Physiol., 269:H571-H582, 1995.

[42] D. A. Hooks, M. L. Trew, B. J. Caldwell, G. B. Sands, I. J. LeGrice, and B. H. Smaill. Laminar arrangement of ventricular myocytes influences electrical behavior of the heart. Circ. Res., 101:e103-e112, 2007.

[43] B. J. Caldwell, M. L. Trew, G. B. Sands, D. A. Hooks, I. J. LeGrice, and B. H. Smaill. Three distinct directions of intramural activation reveal nonuniform side-to-side electrical coupling of ventricular myocytes. Circ. Arrhythm. Electrophysiol., 2:433-440, 2009.

[44] P. A. Helm, H. J. Tseng, L. Younes, E. R. McVeigh, and R. L. Winslow. Ex vivo 3d diffusion tensor imaging and quantification of cardiac laminar structure. Magn. Reson. Med., 54:850$859,2005$.

[45] J. P. Sun, W. J. Stewart, X. S. Yang, R. O. Donnell, A. R. Leon, J. M. Felner, J. D. Thomas, and J. D. Merlino. Differentiation of hypertrophic cardiomyopathy and cardiac amyloidosis from other causes of ventricular wall thickening by two-dimensional strain imaging echocardiography. Am. J. Cardiol., 103:411-415, 2009.

[46] H. Ashikaga, B. A. Coppola, K. G. Yamazaki, F. J. Villarreal, J. H. Omens, and J. W. Covell. Changes in regional myocardial volume during the cardiac cycle: implications for transmural blood flow and cardiac structure. Am. J. Physiol. Heart Circ. Physiol., 295:H610-H618, 2008.

[47] M. J. Bishop, G. Plank, R. A. Burton, J. E. Schneider, D. J. Gavaghan, V. Grau, and P. Kohl. Development of an anatomically detailed MRI-derived rabbit ventricular model and assessment of its impact on simulations of electrophysiological function. Am. J. Physiol. Heart Circ. Physiol., 298:H699-H718, 2010.

[48] S. Niederer, L. Mitchell, N. Smith, and G. Plank. Simulating a human heart beat with nearreal time performance. Front. Physio., 2:1-7, 2011.

[49] N. A. Trayanova and J. J. Rice. Cardiac electromechanical models: from cell to organ. Front. Physiol., 2:43, 2011. 
[50] K. Shinmura, X. L. Tang, Y. Wang, Y. T. Xuan, S. Q. Liu, H. Takano, A. Bhatnagar, and R. Bolli. Cyclooxygenase-2 mediates the cardioprotective effects of the late phase of ischemic preconditioning in conscious rabbits. PNAS, 97:10197-10202, 2000.

[51] J. Stypmann, M. A. Engelen, A. K. Breithardt, P. Milberg, M. Rothenburger, O. A. Breithardt, G. Breithardt, L. Eckardt, and P. N. Cordula. Doppler echocardiography and tissue Doppler imaging in the healthy rabbit: differences of cardiac function during awake and anaesthetised examination. Int. J. Cardiol., 115:164-170, 2007. 


\begin{tabular}{lll} 
Passive stress & Active stress & Pressure term \\
\hline$\mu_{\mathrm{K}}=3333 \mathrm{kPa}$ & $k_{S_{\mathrm{a}}}=0.50 \mathrm{kPa} \mathrm{mV}^{-1}$ & $C=0.2 \mathrm{ml} \mathrm{mmHg}^{-1}$ \\
$a=0.333 \mathrm{kPa}$ & $V_{\mathrm{r}}=-86.796 \mathrm{mV}$ & $R=750 \mathrm{mmHg} \mathrm{ms} \mathrm{ml}^{-1}$ \\
$b=9.242(-)$ & $V_{\mathrm{s}}=-80.0 \mathrm{mV}$ & $C_{\mathrm{p}}=-900 \mathrm{mmHg} \mathrm{ml}^{-1}$ \\
$a_{\mathrm{f}}=18.535 \mathrm{kPa}$ & $\epsilon_{0}=1.0 \mathrm{~ms}^{-1}$ & \\
$b_{\mathrm{f}}=15.972(-)$ & $\epsilon_{\infty}=0.1 \mathrm{~ms}^{-1}$ & \\
$a_{\mathrm{s}}=2.564 \mathrm{kPa}$ & $\zeta_{\mathrm{r}}=0.1 \mathrm{mV}^{-1}$ & \\
$b_{\mathrm{s}}=10.446(-)$ & & \\
$a_{\mathrm{fs}}=0.417 \mathrm{kPa}$ & & \\
$b_{\mathrm{fs}}=11.602(-)$ & & \\
\hline
\end{tabular}

Table 1: Material parameters used in both analytical and numerical calculations. The parameters for the passive state are adapted from [18], the parameters for the active state are adapted from [24], and the pressure term is chosen to give a realistic pressure-volume response. 


\begin{tabular}{clll} 
BC & \multicolumn{2}{c}{ Coordinates } & Description \\
\hline$t_{\mathrm{n}}=-p$ & $\xi_{1}=\xi_{1 \mathrm{~min}}$ & for all $\xi_{2}, \xi_{3}$ & Endocardial surface \\
$u_{\xi_{2}}=0$ & $\xi_{2}=\xi_{2 \max }$ & for all $\xi_{1}, \xi_{3}$ & Basal surface \\
& & & Outer boundary at \\
$u_{\xi_{3}}=0$ & $\begin{array}{l}\xi_{1}=\xi_{1 \max } \\
\xi_{2}=\xi_{2 \max }\end{array}$ & for all $\xi_{3}$ & \begin{tabular}{l} 
the basal surface \\
\hline
\end{tabular} \\
\hline
\end{tabular}

Table 2: Mechanical boundary condition (BC) for the LV in terms of prescribed traction $\mathbf{t}$ and displacement $\mathbf{u}$. The component $t_{\mathrm{n}}$ of $\mathbf{t}$ is the normal to the endocardial surface on which the pressure $p$ acts. The components $u_{\xi_{1}}, u_{\xi_{2}}, u_{\xi_{3}}$ of $\mathbf{u}$ are the displacements in the directions $\xi_{1}, \xi_{2}$, $\xi_{3}$ shown in Figure 2, where the index max and min denotes the maximum and minimum possible coordinate in the respective direction. 


\begin{tabular}{lrrr} 
Result & Model I & Model II & Experiment \\
\hline$h_{\mathrm{f}}(\%)$ & 33 & 29 & $35.2^{\mathrm{a}}$ \\
$V_{\mathrm{f}}^{\max }(\%)$ & -0.42 & -0.51 & $-7.2^{\mathrm{b}}$ \\
$\mathrm{HR}(\mathrm{bmp})$ & 171 & 171 & $198^{\mathrm{c}}$ \\
$\mathrm{SV}(\mathrm{ml})$ & 2.7 & 3.1 & $1.8^{\mathrm{c}}$ \\
$\mathrm{EF}(\%)$ & 61 & 63 & $53^{\mathrm{c}}$ \\
$\mathrm{CO}(1 / \mathrm{min})$ & 0.46 & 0.53 & $0.421^{\mathrm{c}}$ \\
\hline
\end{tabular}

Table 3: Comparison of results between the models $I, I I$ and the experimental data: $h_{\mathrm{f}}=$ fractional thickening, $V_{\mathrm{f}}^{\max }=$ largest change in volume fraction of the wall, $\mathrm{HR}=$ heart rate, $\mathrm{SV}=$ stroke volume, $\mathrm{EF}=$ ejection fraction, $\mathrm{CO}=$ cardiac output. Experimental values are taken from: ${ }^{\mathrm{a}}$ [50]; ${ }^{b}$ average value of the through-the-thickness volume change [46]; ${ }^{c}$ [51]. 


\begin{tabular}{cccccc} 
Case (i) & Case (ii) & Case (iii) & Case (iv) & Case (v) & Case (vi) \\
\hline $51^{\circ}$ & $72^{\circ}$ & $35^{\circ}$ & $47^{\circ}$ & $62^{\circ}$ & $33^{\circ}$ \\
\hline
\end{tabular}

Table 4: Resulting torsion using the cases as outlined in Section 2.3.1. 
Figure 1: (a) Transmembrane potential $V_{\mathrm{m}}$, dotted curve, and corresponding delay in the active stress value using $\epsilon_{\infty}>\epsilon_{0}$, dash-dotted curve, and $\epsilon_{\infty}<\epsilon_{0}$, solid curve; for clarity the values are scaled in the image; (b) shape of the delay function $\epsilon\left(V_{\mathrm{m}}\right)$ using $\epsilon_{\infty}>\epsilon_{0}$, dash-dotted curve, and $\epsilon_{\infty}<\epsilon_{0}$, solid curve, when shifted by $V_{\mathrm{s}}=-30 \mathrm{mV}$ with the parameter $\zeta_{\mathrm{r}}=0.3 \mathrm{mV}^{-1}$.

Figure 2: Ellipsoidal model representing a rabbit LV with both the global $X_{1}, X_{2}$ and $X_{3}$ coordinates and the prolate spheroidal coordinates $\xi_{1}, \xi_{2}$ and $\xi_{3}$; where $a_{\text {endo }}, b_{\text {endo }}, a_{\text {epi }}, b_{\text {epi }}$ are the polar and equatorial axes, and $d_{\text {endo }}$ and $d_{\text {epi }}$ are the focal positions for the endocardial and epicardial borders, respectively. The plane separating the basal and apical regions is half of the LV height $h$. The septal region lies in the $X_{1}, X_{2}$ quadrant, the anterior region lies in the $X_{1},-X_{2}$ quadrant, the lateral region lies in the $-X_{1},-X_{2}$ quadrant and the posterior region lies in the $-X_{1}, X_{2}$ quadrant. The fiber and sheet directions (Lagrangian form) are characterized by $\mathbf{f}_{0}$ and $\mathbf{s}_{0}$, respectively. The inclination angle, measured in the $\left(\xi_{2}, \xi_{3}\right)$ plane, and the sheet angle, measured in the $\left(\xi_{1}, \xi_{2}\right)$ plane, are characterized by $\alpha$ and $\beta$, respectively.

Figure 3: Fiber and sheet angles through the wall thickness of the LV starting from the endocardium, adapted from [2].

Figure 4: (a) Deformation of a unit cube before deformation and after activation in the fiber direction $(X)$, shown in gray; (b) stretch responses as a function of the membrane potential $V_{\mathrm{m}}$. Fiber, sheet and sheet-normal stretches are illustrated by solid, dashed and dotted curves, respectively, and the circles show the corresponding finite element results.

Figure 5: (a) Comparison of pressure-volume (PV) loops between model $I I$ and experimental data [37]. Volume transients for this comparison are normalized to account for the difference in the initial LV volumes; (b) comparison of PV loops between the models $I$ and $I I$. As a representative case for model $I$ the fibers and sheets vary linearly between $\alpha_{\text {endo }}=-60^{\circ}, \alpha_{\text {epi }}=+60^{\circ}$ while $\beta_{\text {endo }}=\beta_{\text {epi }}=0^{\circ}$, respectively (case (i), Section 2.3.1).

Figure 6: Geometries at EDV shown by dashed curves, and at ESV shown by solid curves filled with gray areas: (a) the short axis plane is perpendicular to the long axis plane, which is always aligned with the main direction of the septal wall; (b) the outlined epicardial surface viewed from the apex towards the base of the LV. The apex in both EDV and ESV are marked with a circle and show the movement of the apex towards the septal region; (c) torsion at the short axis plane for the model $I I$. The average torsion is $4.5^{\circ}$; (d) torsion at the short axis plane for the model $I$, case (i) (Section 2.3.1). The torsion is the same for all regions and is $51^{\circ}$.

Figure 7: First principal stress $\sigma_{I}$ : (a) in model $I$; (b) in model $I I$. 

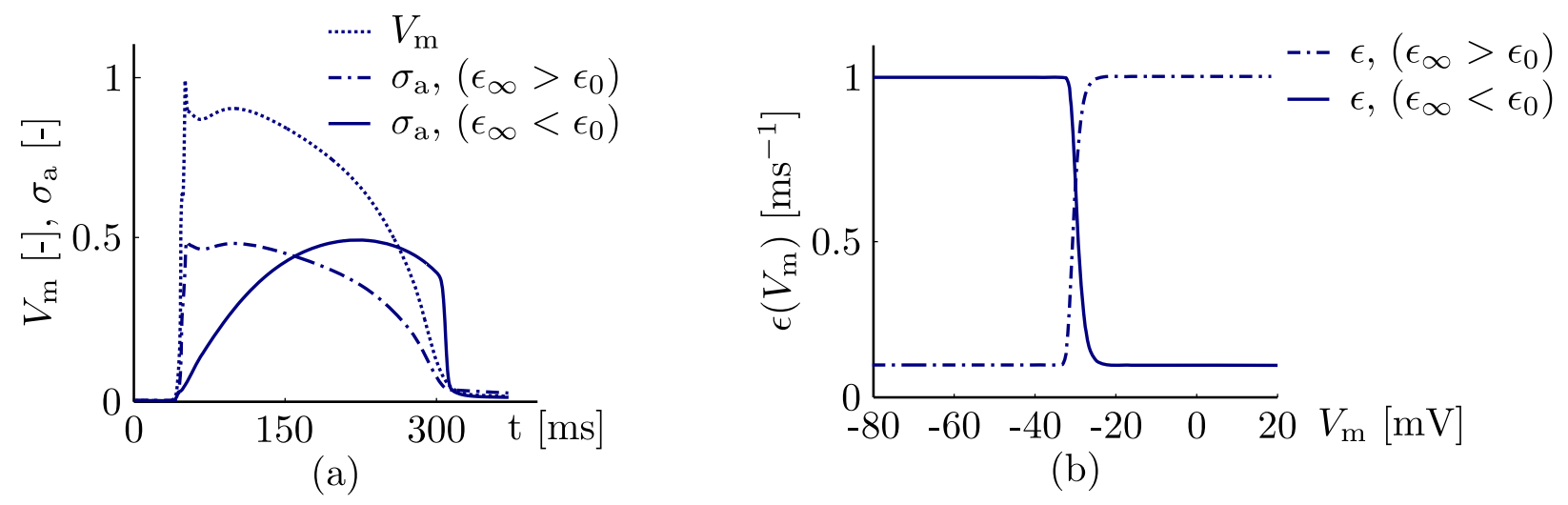

(b)

Figure 1: 


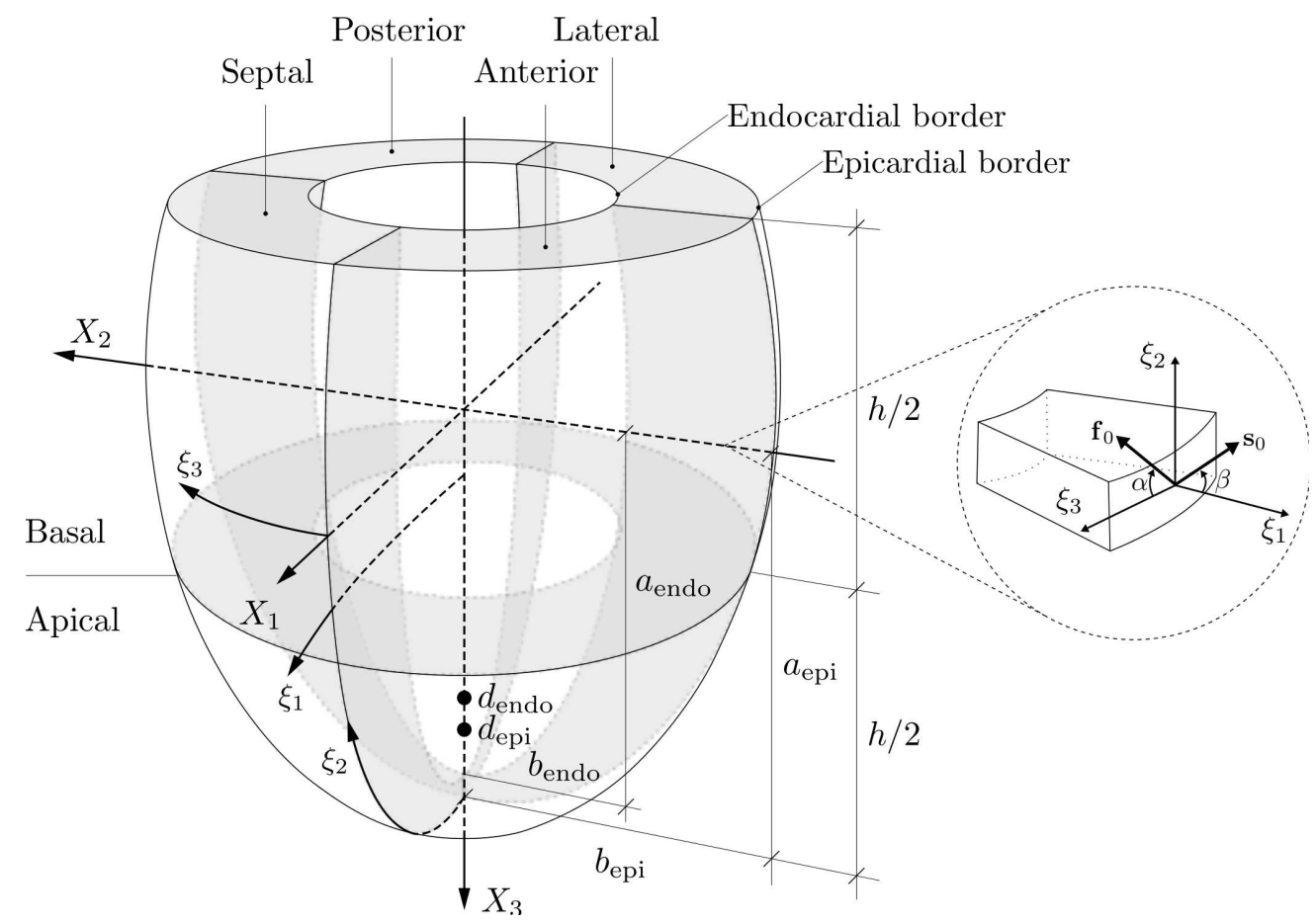

Figure 2: 

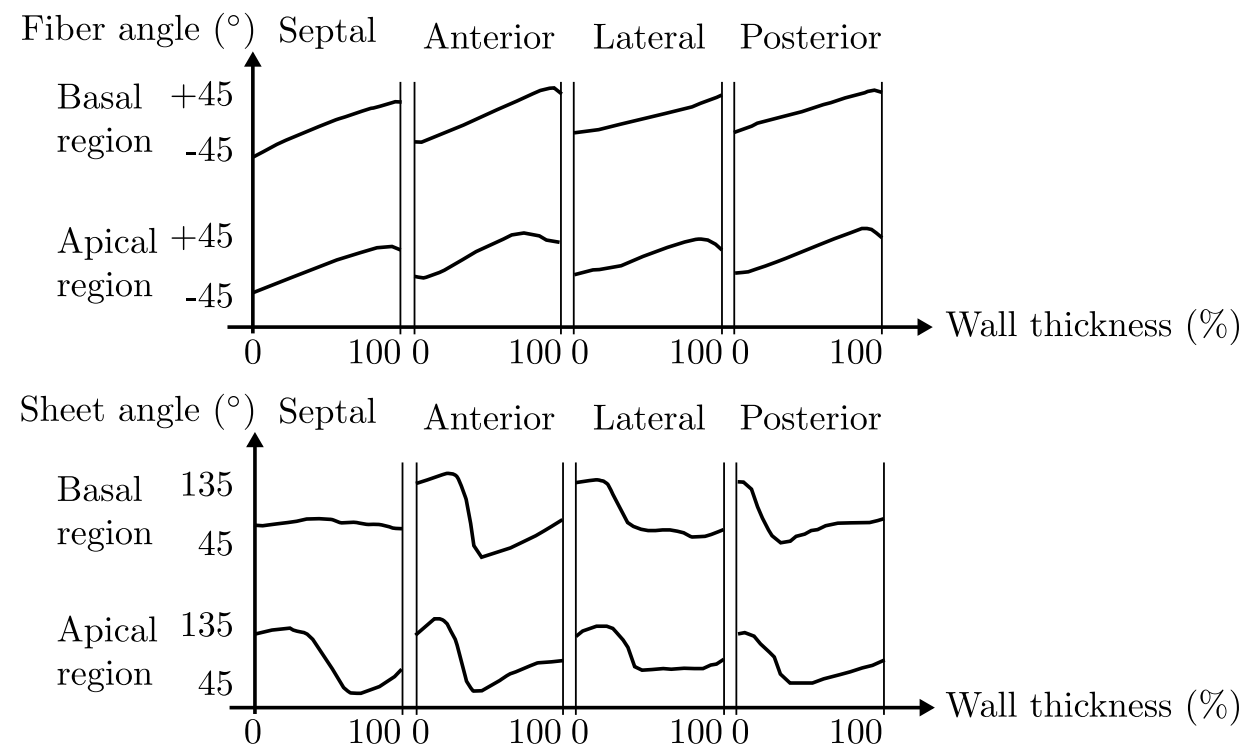

Figure 3: 


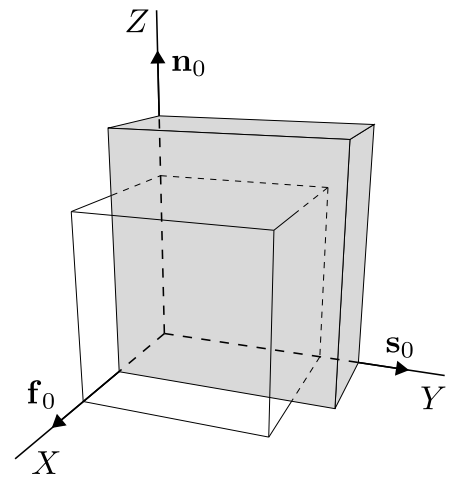

(a)

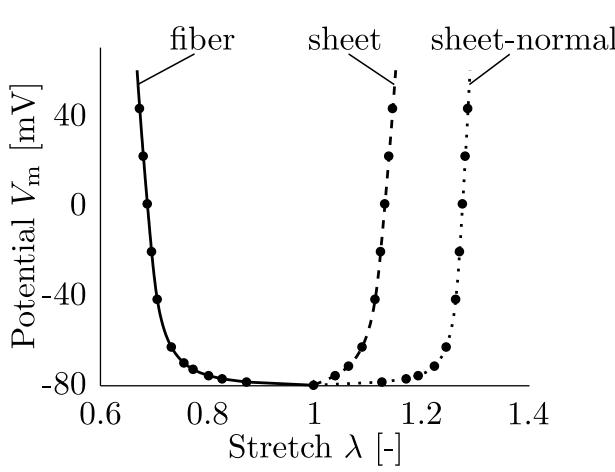

(b)

Figure 4: 


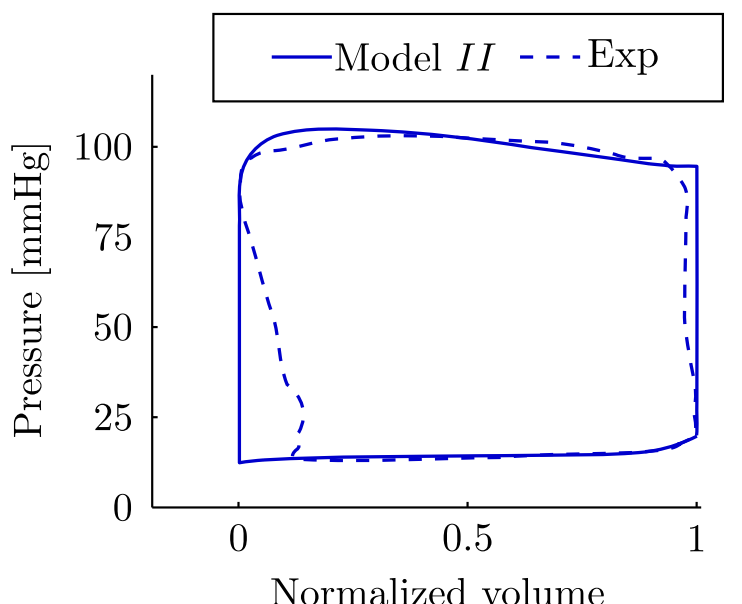

(a)

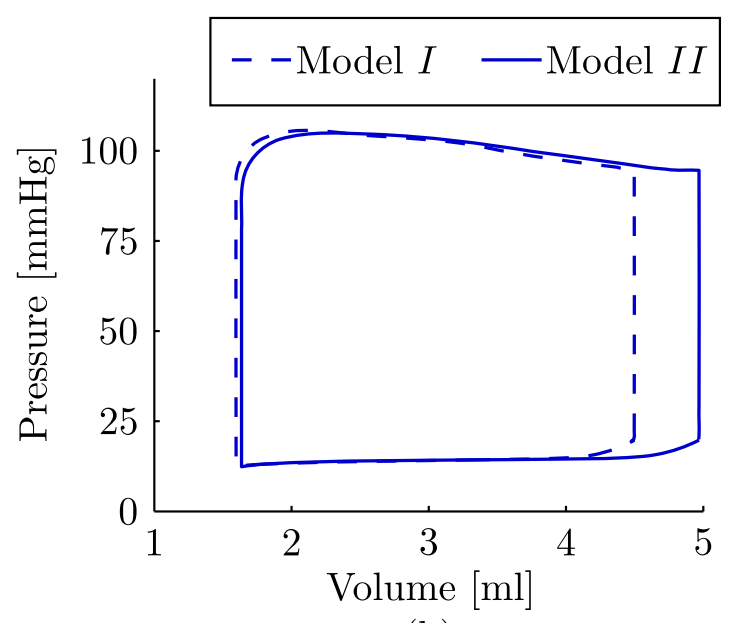

(b)

Figure 5: 


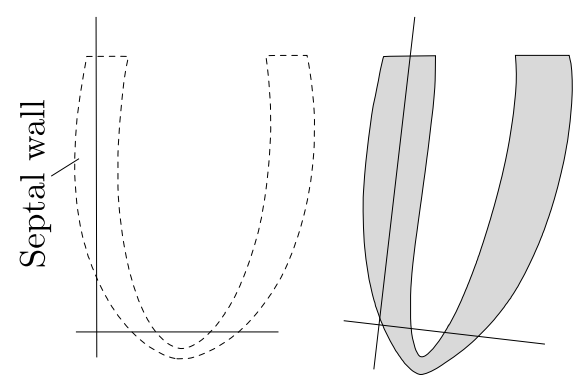

(a)

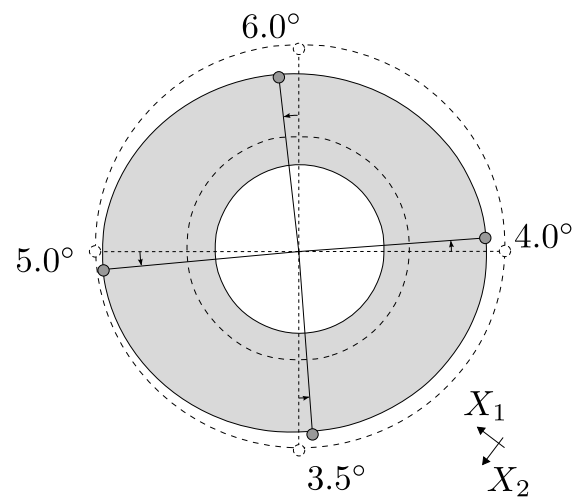

(c)

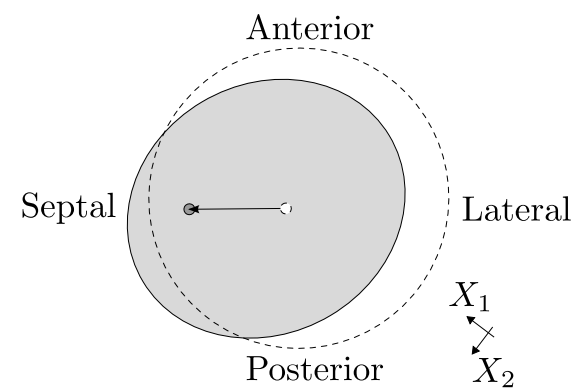

(b)

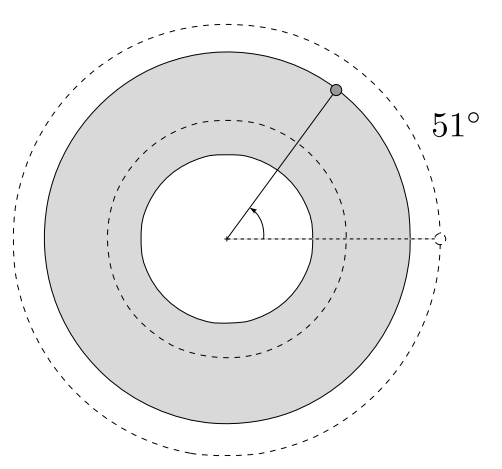

(d)

EDV $\square \mathrm{ESV}$

Figure 6: 


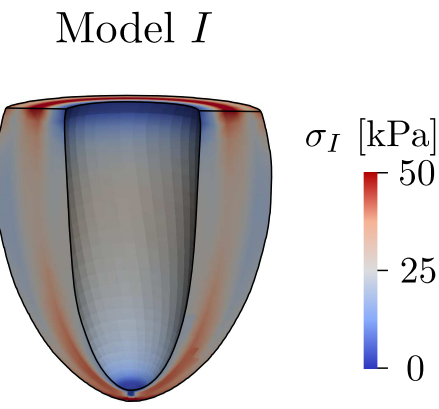

(a)
Model II

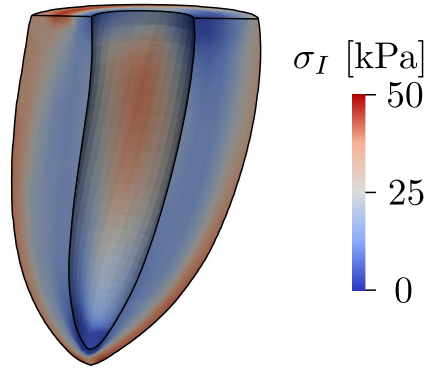

(b)

Figure 7: 